\title{
AUTOMAÇÃO EM SISTEMA DE IRRIGAÇÃO TIPO PIVÔ CENTRAL PARA ECONOMIA DE ENERGIA ELÉTRICA
}

\section{MARIA J. MORAES ${ }^{1}$, DELLY OLIVEIRA FILHO ${ }^{2}$, EVERARDO C. MANTOVANI ${ }^{3}$, PAULO M. B. MONTEIRO ${ }^{4}$, ANDRÉ L. C. MENDES $^{5}$, JORGE H. A. C. DAMIÃO ${ }^{6}$}

RESUMO: A irrigação é de grande importância para a produção de alimentos, e acredita-se que ela seja responsável por boa parte da produção agrícola mundial, mas é grande consumidora de água doce, principalmente na irrigação por aspersão. O objetivo desta pesquisa é avaliar a redução do custo da energia elétrica para irrigação, por meio de sistema automatizado de controle de velocidade em pivô central instalado em encosta. Para tal, foi dimensionado um pivô central com raio de 56,4 metros com 10 emissores com pressão de serviço de 137,8 kPa, representando uma área irrigada de 1,3 ha, e a lâmina bruta de irrigação adotada foi de $10 \mathrm{~mm} \mathrm{dia}^{-1}$. Posteriormente, realizaram-se cálculos para determinar as pressões em cada emissor, para as condições de 0; 10; 20 e 30\% de declividade média do terreno. Posteriormente, foi montado um protótipo do pivô central em tamanho reduzido, com 6,3 metros de comprimento, com 10 emissores. Foi utilizado um conjunto motobomba de 2,21 kW (3cv), onde as perdas de cargas em cada emissor eram realizadas por meio de registros inseridos na linha lateral. A automação e o controle de velocidade implementada no protótipo possibilitaram a redução dos gastos em energia elétrica entre 18\% e 52\%. A automação desenvolvida mostrou-se viável e capaz de responder com agilidade a qualquer mudança de pressão.

PALAVRAS-CHAVE: motores elétricos, bombas, automação em pivô central.

\section{AUTOMATION ON CENTRAL PIVOT IRRIGATION SYSTEM TO ENERGY SAVINGS}

ABSTRACT: Irrigation is of great importance to food production, it is believed that it is responsible for much of the world agricultural production, but appears as a villain in the consumption of fresh water, particularly the sprinkler irrigation. The objective of this research was to evaluate the reduction in the electricity costs for irrigation using automated speed control in central pivot irrigation systems, installed in a slope. For this research, a central pivot was dimensioned with a radius of $56.4 \mathrm{~m}, 10$ emitters, pressure of $137.8 \mathrm{kPa}$, with a irrigated area of 1.3 ha and an the adopted irrigation depth was $10 \mathrm{~mm}^{-1}$. Afterwards, calculations were performed to determine the pressures at each emitter to the conditions of $0,10,20$ and $30 \%$ of average land slope. A mathematical modeling was done, where the hydraulic calculations were made for these conditions. It was later mounted a prototype central pivot in small size with 6.3 long with 10emitters. It was used a motor pump set with $2.21 \mathrm{~kW}(3 \mathrm{hp})$, and the pressure drop in each emitter was done by adding flow controller on the lateral line. The automation and speed control implemented in the prototype enabled the reduction of electricity costs from $18 \%$ to $52 \%$. The automation developed show to be viable and able to respond quickly to any pressure change.

KEY WORDS: Electric motors, pumps, demand side management.

\footnotetext{
${ }^{1}$ Eng a Agrícola, Profa. Dra Unidade de Ciências Exatas e Tecnológicas Uni. Estadual de Goiás Anapolis-GO, maria.moraes@ufv.br

${ }^{2}$ Eng $^{\circ}$ Eletricista, Prof. Dr. Dpto. de Eng. Agrícola, Uni. Federal de Viçosa, Viçosa/MG, delly@ufv.br.

${ }^{3}$ Eng $^{\circ}$ Agrícola, Prof. Titular Depto. de Eng. Agrícola, Uni. Federal de Viçosa, Viçosa/MG, everardo@ufv.br.

${ }^{4}$ Eng $^{\circ}$ Eletrônico, Prof. Dr. Depto. de Eng. de Controle e Automação, Uni. Federal de Ouro Preto, paulo@em.ufop.br.

${ }^{5}$ Eng $^{0}$ Eletricista, M.Sc. Uni. Federal de Viçosa, andre.mendes@ufv.br

${ }^{6}$ Eng $^{\circ}$ Agrícola e Ambiental, Distrito de Irrigação do Perímetro Gorutuba, Nova Porteirinha/MG, damiaojh@gmail.com

Recebido pelo Conselho Editorial em: 8-5-2013
}

Aprovado pelo Conselho Editorial em: 6-5-2014 


\section{INTRODUÇÃO}

A importância dos recursos naturais para a sobrevivência humana é incontestável, e um dos maiores desafios (tendência/perspectiva) é a produção de alimentos para atender à crescente demanda mundial. Um forte aliado para o aumento da produção é a utilização de tecnologias de irrigação, que ocupa uma área cultivada 4,5 vezes menor que a agricultura de sequeiro e contribui com cerca de metade a dois terços da produção de alimentos ( RIBEIRO, 2008).

A utilização da irrigação tem objetivo de aumentar o lucro, com o aumento da produção e da qualidade, e/ou de incorporar à agricultura áreas que não seriam possíveis de se cultivar sem o uso da irrigação. Mas a irrigação pode encontrar grandes desafios futuros, uma vez que a disponibilidade de água para a irrigação será reduzida, devido ao aumento da demanda por outros setores considerados prioritários (SANTOS et al., 2010; VIEIRA et al., 2011).

Sabe-se, obviamente, que a energia é um dos parâmetros mais importantes tanto para o crescimento econômico quanto para o desenvolvimento social de uma região. Logo, faz-se necessário utilizar, por exemplo, previsões científicas e análises de consumo, no planejamento de estratégias e políticas do uso de energia. No cenário atual, o uso de energia na agricultura tem sido intensificado em resposta ao contínuo crescimento populacional, bem como a oferta limitada de terra agricultável. Por consequência, os agricultores usam seus insumos em excesso e de forma ineficiente, especialmente quando esses têm preço baixo ou estão disponíveis em abundância. Percebe-se, assim, que a melhoria da eficiência energética não só ajuda a melhorar a competitividade, através da redução de custos, como também minimiza a poluição ambiental relacionada à energia (MOUSAVI-AVVAL et al., 2011).

Segundo Moreno et al. 2010, entre os métodos de irrigação mais difundidos atualmente no mundo, estão os sistemas de irrigação por aspersão convencional e pivô central. Sendo esse último, em muitos casos, empregado sem critérios necessários para que se possa obter o máximo de rendimento de uso do recurso água e energia elétrica. O uso de água em excesso eleva o gasto com energia elétrica, que pode chegar a $25 \%$ dos custos de produção, diminuindo a renda do produtor.

O gasto excessivo de energia pode ser causado por problemas, como: (i) procedimentos operacionais; (ii) falhas do dimensionamento técnico; (iii) deficiência da manutenção preventiva ou corretiva; (iv) baixa eficiência dos equipamentos elétricos ou hidráulicos, (v) contratos de energia elétrica inadequados; e (vi) lâmina d'água aplicada inadequadamente.

JIMÉNEZ-BELLO et al., (2011) listam algumas medidas que podem ser adotadas para reduzir o consumo de energia durante a operação de sistemas de irrigação: (i) a determinação do diâmetro ótimo do tubo, após o estudo da rede, levando em conta o critério de energia; (ii) a seleção da estação de bombeamento, considerando a demanda de água prevista; (iii) a administração eficiente conseguida através de protocolos e ferramentas que avaliam o desempenho, usando indicadores de gestão.

Importantes ganhos econômicos foram observados na Espanha após terem sido realizadas auditorias energéticas, resultando em maior eficiência energética dos sistemas de irrigação como um todo (MORENO et al, 2010).

Em processos onde se exige controle de velocidade, a utilização de inversores de frequência é extremamente indicada, sendo já muito utilizado na indústria e está ganhando espaço na agricultura, além de controlar a velocidade o inversor de frequência empregado em sistemas de irrigação trás outros benefícios como: adequação de carga; correção do fator de potência; partidas suaves; redução de perdas nas válvulas; maior flexibilidade de operação; melhor controle sobre parâmetros do processo, e melhoria e conforto das condições operacionais pelo menor nível de ruído.

O sistema de irrigação por aspersão do tipo pivô central com inversores de frequência pode ser atraente economicamente e, do ponto de vista operacional, exigir menos mão de obra e ação de controle mais precisa. Quanto ao controle do volume da água aplicada na irrigação, estudos 
comprovam que o funcionamento de sistemas de bombeamento sob rotação nominal permite menor flexibilidade no manejo do que aqueles sob regime de rotação variável. No entanto, a rotação deverá ser sempre ajustada em função do ponto de estado almejado (OLIVEIRA FILHO et al., 2011,

Sistemas automatizados podem ser utilizados para o controle dos sistemas de irrigação, visando à economia de energia e de água quando houver a necessidade de variação da vazão. Segundo FERNANDES \& TESTEZLAF, (2002), sistemas automatizados de controle de irrigação tornam-se uma ferramenta essencial para a aplicação de água na quantidade necessária e no devido tempo, contribuindo para o aumento da produção e a diminuição do gasto de produção.

SUDHA et al. (2011) utilizaram comunicação sem fio wireless em um sistema de irrigação, testando 2 tipos de protocolos, e conseguiu redução significativa no consumo de energia elétrica.

RIBEIRO, (2008) propôs-se à construção de um protótipo de pivô central em escala reduzida e seu sistema de bombeamento acoplado ao inversor de frequência acionado manualmente, com o objetivo de racionalização de água e energia em sistemas de irrigação por pivô central, alcançando economia de energia elétrica da ordem de 26 \% para inclinação máxima de $20 \%$ da linha lateral do pivô central.

Com o exposto anteriormente, objetivou-se com esta pesquisa avaliar a redução do custo da energia elétrica para irrigação, com o uso de inversores de frequência para automação de sistemas.

\section{MATERIAL E MÉTODOS}

Este experimento foi realizado no Laboratório de Hidráulica da Universidade Federal de Viçosa, Viçosa-MG. Foi montando em laboratório um protótipo de um pivô central, seguindo a metodologia de BERNARDO et al., (2006), com uma área equivalente total circular irrigada de 1,03 ha. No dimensionamento do pivô, foi usada uma lâmina bruta diária de $10 \mathrm{~mm}$ e tempo de 14 horas para uma volta completa, o espaçamento entre emissores foi de 4,6 m, e os emissores selecionados foram do modelo Asfix da marca Fabrimar, com diâmetro molhado médio de 8 m.

\section{Dimensionamento do pivô central}

Foi realizado o dimensionamento de um pivô central com área de 1,03 ha, para uma lâmina de irrigação bruta de $10 \mathrm{~mm} /$ dia, com pressão de serviço dos aspersores de 137, $2 \mathrm{kPa}$. Foi dimensionado um sistema de irrigação por pivô central usando a metodologia de BERNARDO et al., (2006). [eq. (1)],

Para o cálculo da perda de carga nas tubulações, foi utilizada a equação de Hazen-Williams

$$
h_{f}=10,643 \frac{L Q^{1,852}}{D^{4,853} C^{1,852}}
$$

em que:

$h_{f} \quad$ - Perda de carga, mca;

$Q \quad$ - Vazão, $\mathrm{m}^{3} \mathrm{~s}^{-1}$;

D - Diâmetro interno da tubulação, m;

$L \quad$ - Comprimento, m;

C - Coeficiente de rugosidade do tubo; $\mathrm{C}(\mathrm{pvc})=135$.

A potência hidráulica e a potência absorvida pela bomba foram definidas de acordo com as eqs. (2) e (3) (RIBEIRO, 2008), 


$$
P_{a}=\frac{\gamma Q H_{\operatorname{man}}}{75 \eta_{b}}
$$

em que,

$P_{u}$ - Potência hidráulica ou útil, kW;

$P_{a}$ - Potência absorvida pela bomba, kW;

$\gamma$ - Peso específico da água, $\left(\mathrm{Nm}^{-3}\right)$;

$Q$ - Vazão, $\mathrm{L} \mathrm{s}^{-1}$;

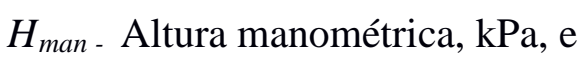

$\eta_{b}$ - Rendimento da bomba, em decimal.

Para a análise da potência elétrica utilizada na variação da inclinação da lateral do pivô central, foi utilizada a [eq. (4)], em que a vazão, as alturas manométricas e os rendimentos da bomba e do motor são funções das frequências (VIANA et al., 2004),

$$
P_{e l}=\frac{1000 g Q(f)\left(H_{2}(f)-H_{1}(f)\right)}{\eta_{b}(f) \eta_{m}(f)}
$$

em que,

$P_{e l}$ - Potência elétrica, $\mathrm{kW}$;

$g$ - Aceleração da gravidade, $\mathrm{m} \mathrm{s}^{-2}$;

$Q$ - Vazão, $\mathrm{m}^{3} \mathrm{~s}^{-1}$;

$H_{1}$ - Altura manométrica inicial, kPa;

$\mathrm{H}_{2}$ - Altura manométrica final, $\mathrm{kPa}$;

$\eta_{b}$ - Rendimento da bomba, em decimal;

$\eta_{m}$ - Rendimento do motor, em decimal, e

$f$ - Frequência, Hz.

O índice de carregamento de um motor elétrico é definido como a razão entre a potência demandada e a potência nominal, de acordo com a [eq. (5)],

$$
I_{c}=\frac{P_{a}}{P_{n}} 100
$$

em que,

$I_{C}$ Índice de carregamento, \%;

$P_{a}$ Potência absorvida pela bomba, $\mathrm{kW}$, e

$P_{n}$ Potência nominal do motor, kW.

Para o dimensionamento do protótipo do pivô central, utilizou-se de uma lâmina de irrigação de $10 \mathrm{~mm} \mathrm{dia}^{-1}$, com espaçamento entre emissores de 4,6 m, formando um diâmetro médio de alcance dos emissores de $8 \mathrm{~m}$ (Tabela 1). O comprimento total da linha lateral do pivô foi de 54,6 m, formando uma área irrigada de 1,03 hectares, para um tempo de irrigação de 14 horas. 
TABELA 1. Caracterização do pivô central para diâmetro médio de alcance dos emissores de 8 m, para lâmina de irrigação de $10 \mathrm{~mm} \mathrm{dia}^{-1}$. Characterization of central pivot for diameter range of $8 \mathrm{~m}$ emitters for irrigation depth of $10 \mathrm{~mm} \mathrm{day}^{-1}$

\begin{tabular}{cccccccccc}
\hline Vazão & $\begin{array}{c}\text { Vazão } \\
\text { Bocal }\end{array}$ & $\begin{array}{c}\text { Diâm. } \\
\text { Bocal }\end{array}$ & $\begin{array}{c}\text { Esp. Entre Emist. } \\
\text { Emissores }\end{array}$ & $\begin{array}{c}\text { Eixo do } \\
\text { pivô }\end{array}$ & $\begin{array}{c}\text { Área } \\
\text { irrigada }\end{array}$ & $\begin{array}{c}\text { Tempo } \\
\text { irrigação }\end{array}$ & $\begin{array}{c}\text { Tem/ } \\
\text { volta }\end{array}$ & $\begin{array}{c}\text { Lâmina } \\
\text { média }\end{array}$ & $\begin{array}{c}\text { Irrigação } \\
\text { Média }\end{array}$ \\
\hline$\left(\mathrm{m}^{3} \mathrm{~h}^{-1}\right)$ & $\left(\mathrm{m}^{3} \mathrm{~h}^{-1}\right)$ & $(\mathrm{mm})$ & $(\mathrm{m})$ & $(\mathrm{m})$ & $\left(\mathrm{m}^{2}\right)$ & $(\mathrm{min})$ & $(\mathrm{h})$ & $(\mathrm{mm} / \mathrm{volta})$ & $(\mathrm{mm} / \mathrm{h})$ \\
\hline 0,31 & 0,35 & 2,8 & 15 & 15 & 433,5 & 71,3 & 14 & 11,3 & 9,5 \\
\hline 0,4 & 0,4 & 3,0 & 4,6 & 19,6 & 566,4 & 54,6 & 14 & 9,9 & 10,9 \\
\hline 0,5 & 0,51 & 3,4 & 4,6 & 24,2 & 699,4 & 44,2 & 14 & 10,2 & 13,9 \\
\hline 0,59 & 0,64 & 3,8 & 4,6 & 28,8 & 832,4 & 37,1 & 14 & 10,8 & 17,4 \\
\hline 0,69 & 0,71 & 4,0 & 4,6 & 33,4 & 965,3 & 32 & 14 & 10,3 & 19,3 \\
\hline 0,78 & 0,78 & 4,2 & 4,6 & 38 & $1.098,3$ & 28,1 & 14 & 9,9 & 21,2 \\
\hline 0,88 & 0,93 & 4,6 & 4,6 & 42,6 & $1.231,2$ & 25,1 & 14 & 10,6 & 25,3 \\
\hline 0,97 & 1,01 & 4,8 & 4,6 & 47,2 & $1.364,2$ & 22,7 & 14 & 10,4 & 27,5 \\
\hline 1,07 & 1,11 & 5,0 & 4,6 & 51,8 & $1.497,1$ & 20,6 & 14 & 10,4 & 30,2 \\
\hline 1,16 & 1,19 & 5,2 & 4,6 & 56,4 & $1.630,1$ & 19 & 14 & 10,2 & 32,3 \\
\hline- & 7,63 & Total & - & - & $10.318,2$ & - & - & & - \\
\hline
\end{tabular}

Posteriormente, foi reproduzido o protótipo desenvolvido por RIBEIRO, (2008) com algumas modificações, sendo implementado um sistema de controle automático de velocidade de rotação do conjunto motobomba, utilizando inversor de frequência.

O mesmo foi montado no Laboratório de Hidráulica do Departamento de Engenharia Agrícola (DEA) da Universidade Federal de Viçosa, em Viçosa-MG, que serviu de validação do modelo. Para esta montagem, foram utilizados emissores difusores da marca Fabrimar. A linha de irrigação foi montada em escala reduzida, em um protótipo de pivô central (Figura 1), em que: (1) fonte de energia elétrica trifásica, $220 \mathrm{~V}$; (2) inversor de frequência YASKAWA, modelo VS-616; (3) motor elétrico trifásico de 2,2 kW (3 cv), $3.460 \mathrm{rpm}, 2$ polos e $60 \mathrm{~Hz}$; (4) bomba hidráulica - Schneider modelo ME 2230, 2,2 kW, 2 estágios e diâmetro de rotor de $129 \mathrm{~mm}$, que fornece uma altura manométrica na faixa de $10-590 \mathrm{kPa}$ e vazão de $14,9-4,7 \mathrm{~m}^{3} \mathrm{~h}^{-1}$;(5) tubo de PVC, DN $50 \mathrm{~mm}$; (6) ponto de tomada de pressão no início da linha de irrigação (Pin); (7) válvula de gaveta de 50 mm; (8) ponto de tomada de pressão na linha de irrigação - manômetro; (9) derivação de 50 mm para 20 mm; (10) registro de esfera de 1/2” ; (11) ponto de tomada de pressão - manômetro; (12) hidrômetro de 1/2" com conexão para 3/4"; (13) válvula reguladora de pressão, 138,18 kPa (20 PSI); e (14) emissores Fabrimar - vazão de 0,35 a 1,19 $\mathrm{m}^{3} \mathrm{~h}^{-1}$.

Foram avaliados os índices de carregamento do motor elétrico para verificar o respectivo potencial de redução de energia elétrica para cada posição da linha lateral do pivô central; e os valores da altura manométrica e perda de carga obtidos no dimensionamento para cada inclinação foram inseridos no protótipo, a fim de estabelecer condições similares à situação de campo. 


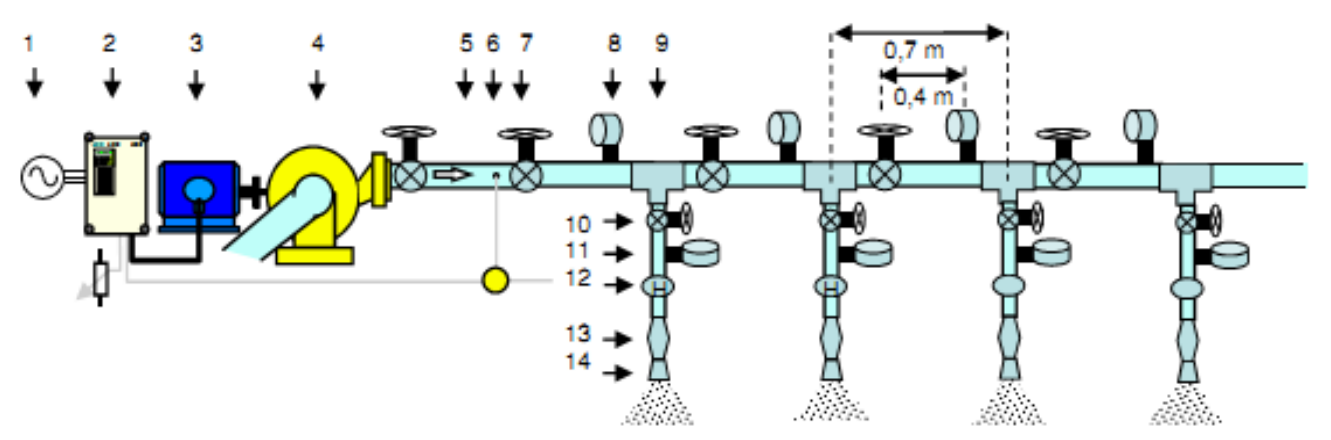

FIGURA 1. Equipamentos e conexões utilizados na construção do protótipo de sistema de irrigação. Equipment and connections used in the construction of the prototype of the irrigation system (RIBEIRO, 2008).

Com isso, foram coletados no protótipo os parâmetros hidráulicos e elétricos para as posições de 0 (em nível); 10; 20 e 30\% (aclive), a fim de manter os limites necessários de pressão e vazão do pivô central. Considerando em $0 \%$ a linha lateral se encontra em nível e 30\%, o aclive máximo correspondendo a $90^{\circ}$ do círculo trigonométrico, a Figura 2 ilustra o croqui da área simulada. Portanto, o protótipo desenvolvido limita-se à simulação de 0 a $180^{\circ}$ de rotação do pivô central real.

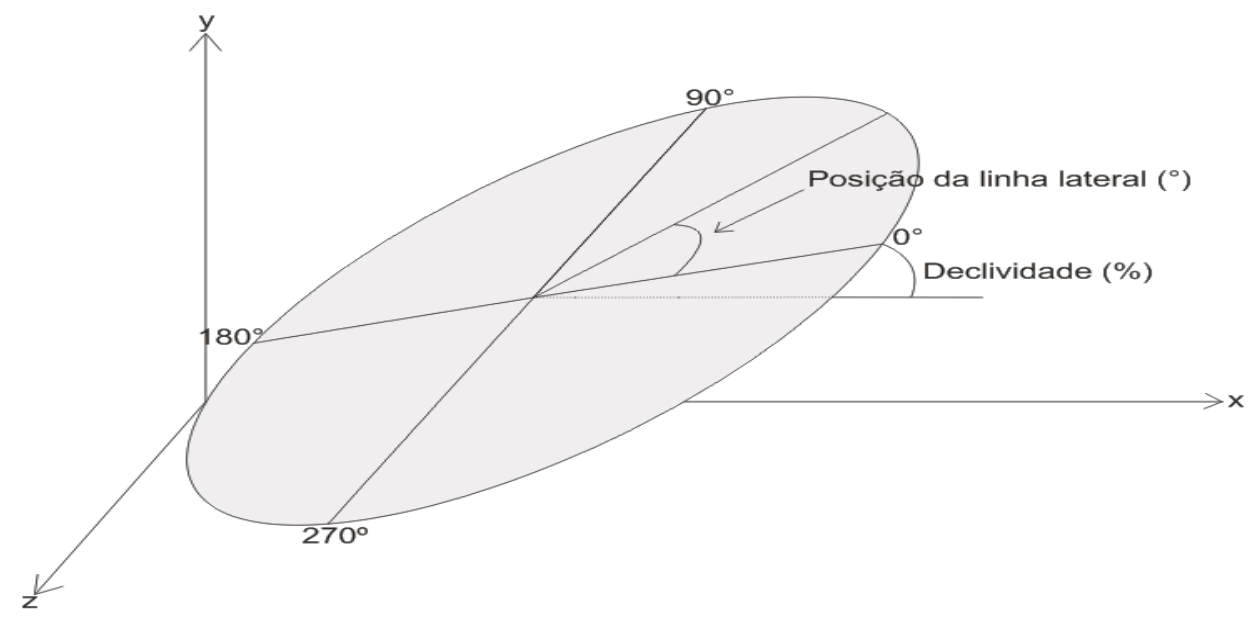

Figura 2. Croqui da área simulada. Sketch of the simulated area.

Para a determinação dos dados elétricos, foi utilizado um sistema de aquisição de dados elétricos, medidor universal de grandezas (MUG) da marca ELO, modelo 2160, instalado no circuito elétrico do motor e programado para adquirir dados por fase da corrente, tensão, potência e fator de potência a cada cinco minutos. Já a rotação do eixo do motor foi medida pelo instrumento Tacômetro TD-700 Instrutherm.

Como o sistema em laboratório permitia somente a simulação de um quarto de volta, então o ponto crítico seria o emissor 10 (e10).

O inversor de frequência não tinha uma comunicação digital, e somente controle de velocidade por meio de tensão de referência. Foi adaptada uma resistência variável acoplada a um motor de passo para que o microcontrolador atuasse no controle de velocidade do conjunto motobomba.

Foi utilizado um microcontrolador da marca microchip, modelo 16f883, com conversor analógico digital interno, transdutor de pressão Motorola MPX5700ap, 15kPa, a $700 \mathrm{kPa}$, e um motor de passo tradicional. O transdutor de pressão foi instalado no ponto crítico, no final da linha lateral, i. e., o $10^{\circ}$ emissor. 
A Figura 3 apresenta um esquema da montagem do sistema em laboratório que representa a irrigação por pivô central, com a interface entre o sistema de bombeamento, o inversor de frequência e a automação.

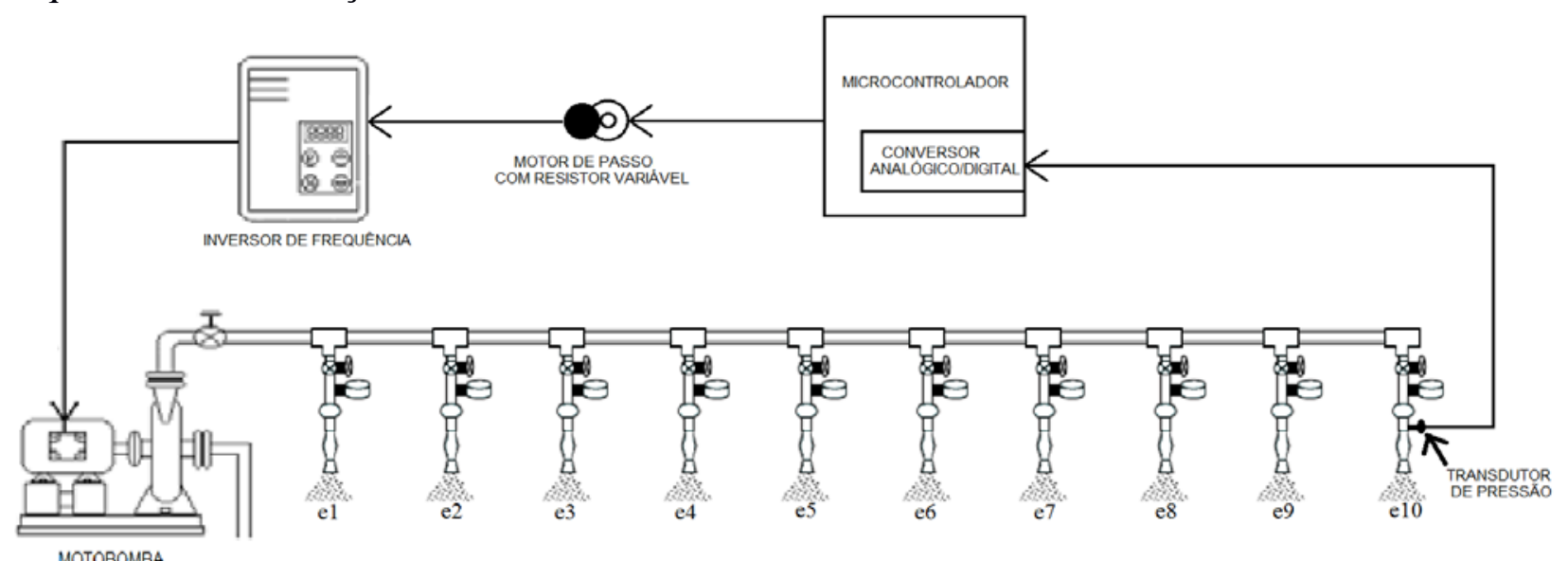

FIGURA 3. Esquema da automação com controle de velocidade em função da pressão no fim da linha lateral. Scheme of automation with speed control depending on the pressure at the end of the sideline.

O microcontrolador recebe o sinal analógico do transdutor de pressão e converte esse sinal em um valor digital. Em seguida, o microcontrolador processa as informações, assim como corrige a pressão de acordo com as equações dos manômetros e calcula o número de acionamentos do motor de passo, para que a pressão no emissor 10 permanecesse acima e, ao mesmo tempo, o mais próximo possível do limite inferior. Depois de terminado o ciclo, o processo repete-se: como ilustrado no fluxograma da Figura 4.

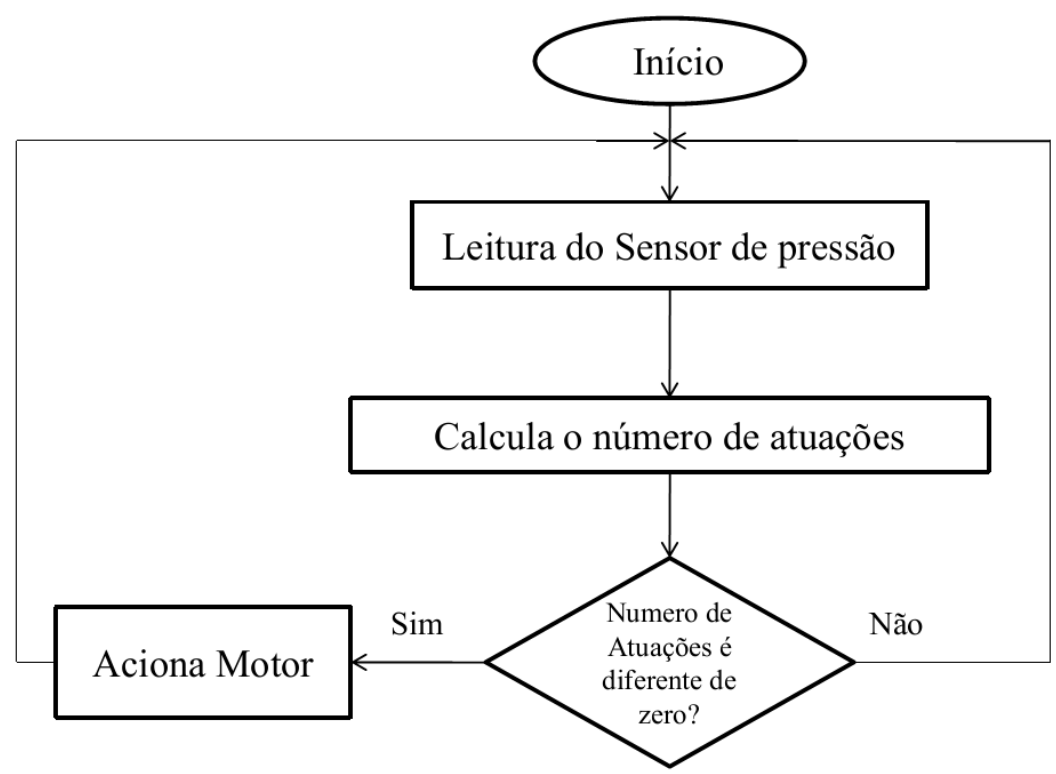

FIGURA 4 - Fluxograma do processo. Process flowchart.

A instalação do protótipo do sistema de irrigação por aspersão tipo pivô central, com o uso de inversor de frequências, foi ajustada por meio de controle da pressão, para simular uma inclinação da linha lateral que possa variar até 30\% (BERNARDO et al., 2006; RIBEIRO, 2008). A simulação do pivô central foi feita com a utilização de tubulação com a regulagem da pressão após a saída para a tubulação do emissor, por meio de válvulas de estrangulamento (válvulas de gaveta). As variações da rotação foram em função da pressão do sistema projetado para cada momento, bem como 
observada a sua vazão, por meio de hidrômetros. Como em RIBEIRO, (2008), foram mantidas as válvulas reguladoras de pressão nos emissores e verificados os limites de suas atuações com e sem o uso do inversor de frequência.

\section{RESULTADOS E DISCUSSÃO}

Para fazer a simulação em laboratório, foi realizada a equivalência do protótipo de 6,3 m para a linha de irrigação de 56,4 m do pivô central, com uma lâmina de irrigação de $10 \mathrm{~mm} \mathrm{dia}^{-1}$. Observa-se que permanecem as mesmas condições de vazão, e consequentemente, os mesmos emissores (Tabela 2).

TABELA 2. Caracterização do protótipo para lâmina de $10 \mathrm{~mm} \mathrm{dia}^{-1}$ para dar uma volta em $14 \mathrm{~h}$ com válvula reguladora de pressão de $137,8 \mathrm{kPa}$. Prototype characterization or an irrigation depth 10 mmday $^{-1}$ for a round in $14 \mathrm{~h}$ with pressure regulating valve of $137.8 \mathrm{kPa}$

\begin{tabular}{cccccccccccc}
\hline Emissor & & 1 & 2 & 3 & 4 & 5 & 6 & 7 & 8 & 9 & 10 \\
\hline Vazão $\left(\mathrm{m}^{3} \mathrm{~h}^{-1}\right)$ & - & 0,35 & 0,40 & 0,51 & 0,64 & 0,71 & 0,78 & 0,93 & 1,01 & 1,11 & 1,19 \\
\hline Trecho & MB-Pin & Pin-1 & $1-2$ & $2-3$ & $3-4$ & $4-5$ & $5-6$ & $6-7$ & $7-8$ & $8-9$ & $9-10$ \\
\hline Distância por trecho $(\mathrm{m})$ & 6 & 15,00 & 4,60 & 4,60 & 4,60 & 4,60 & 4,60 & 4,60 & 4,60 & 4,60 & 4,60 \\
\hline Distância do centro do pivô & 0 & 15,00 & 19,60 & 24,20 & 28,80 & 33,40 & 38,00 & 42,60 & 47,20 & 51,80 & 56,40 \\
\hline
\end{tabular}

A Tabela 3 mostra as pressões e as cotas calculadas para cada emissor para as declividades de 0, 10; 20 e 30\%. Observa-se que o ponto de maior cota é para a declividade de $30 \%$ no emissor 10, considerado este ponto como ponto de projeto, sendo que o mesmo foi usado para o dimensionamento do conjunto motobomba. Este ponto exigirá maior transferência de energia potencial do rotor da bomba para o fluido.

TABELA 3. Pressões e cotas da linha de irrigação para declividades de 0; 10; 20 e 30\%. Pressures and height of the irrigation line to land slopes of $0,10,20$ and $30 \%$

\begin{tabular}{|c|c|c|c|c|c|c|c|c|c|c|c|}
\hline & & & & & \multicolumn{7}{|c|}{ Declividade } \\
\hline & & & & & $0 \%$ & $10 \%$ & $20 \%$ & & $30 \%$ & & \\
\hline \multirow[t]{2}{*}{$\begin{array}{l}\text { Trecho/ } \\
\text { emissor }\end{array}$} & $\begin{array}{c}\text { Compr. do } \\
\text { trecho }\end{array}$ & Vazão & $\begin{array}{l}\mathrm{hf}(*) \\
(\mathrm{m})\end{array}$ & Cota & Pressão & Cota & Pressão & Cota & Pressão & Cota & Press \\
\hline & (m) & $\left(\mathrm{m}^{3} \mathrm{~h}^{-1}\right)$ & & (m) & $(\mathrm{kPa})$ & (m) & $(\mathrm{kPa})$ & (m) & $(\mathrm{kPa})$ & (m) & $(\mathrm{kPa})$ \\
\hline Emissor & 6,00 & 763 & 0,2 & 0,00 & 407,09 & 0,00 & 407,09 & 0,00 & 407,09 & 0,00 & 7,09 \\
\hline 1 & 15, & & 0,5 & 0,0 & 40 & 1,50 & 386,81 & 3,00 & 372,11 & 4,50 & \\
\hline 2 & 4, & & 0,1 & 0,0 & 39 & 1,96 & 380,73 & 3,92 & 361,52 & 5,88 & \\
\hline 3 & 4,60 & 6 & 0,1 & 0,0 & 398 & 2,42 & 374,85 & 4,84 & 351,13 & 7,26 & 327,42 \\
\hline 4 & 4,60 & 6 & 0,1 & 0,0 & 397 & 2,88 & 369,07 & 5,76 & 340,84 & 8,64 & 312,62 \\
\hline 5 & 4 & & 0,1 & 0,0 & 396 & 3,34 & 363 & 6,68 & 330,85 & 10,02 & 29 \\
\hline 6 & 4 & 5 & 0,0 & 0,0 & 395 & 3,80 & 358,29 & 7,60 & 321,05 & 11,40 & 283,81 \\
\hline 7 & 4 & 4 & 0,0 & 0,0 & 394 & 4,26 & 353,19 & 8,52 & 311,44 & 12,78 & 269,70 \\
\hline 8 & & & 0,0 & 0,0 & 394,65 & 4,72 & 348,39 & 9,44 & 302,13 & 14,16 & 255,88 \\
\hline 9 & & & 0,0 & 0,00 & 39 & 5,18 & 343,69 & 10,36 & 292,92 & 15,54 & 242,16 \\
\hline 10 & 4,60 & 1,19 & 0,01 & 0,00 & 394,35 & 5,64 & 333,79 & 11,28 & 283,81 & 16,92 & 228,34 \\
\hline
\end{tabular}

(*) Equação de Hazen Williams (BERNARDO et al., 2006.)

Para atender ao ponto de projeto (declividade de 30\% no emissor 10), foi escolhido um conjunto motobomba, Schneider modelo ME 2230, $2,2 \mathrm{~kW}$, com dois estágios, e diâmetro do rotor de 0,129 metros, fornecendo a vazão de 7,63 $\mathrm{m}^{3} \mathrm{~h}^{-1}$ e a altura manométrica de 436,59 $\mathrm{kPa}$. Observam-se, na Figura 5, as curvas características do conjunto motobomba fornecidas pelo fabricante, e também as curvas características do rendimento do sistema com e sem inversor de frequência, obtidas em ensaio de laboratório. 


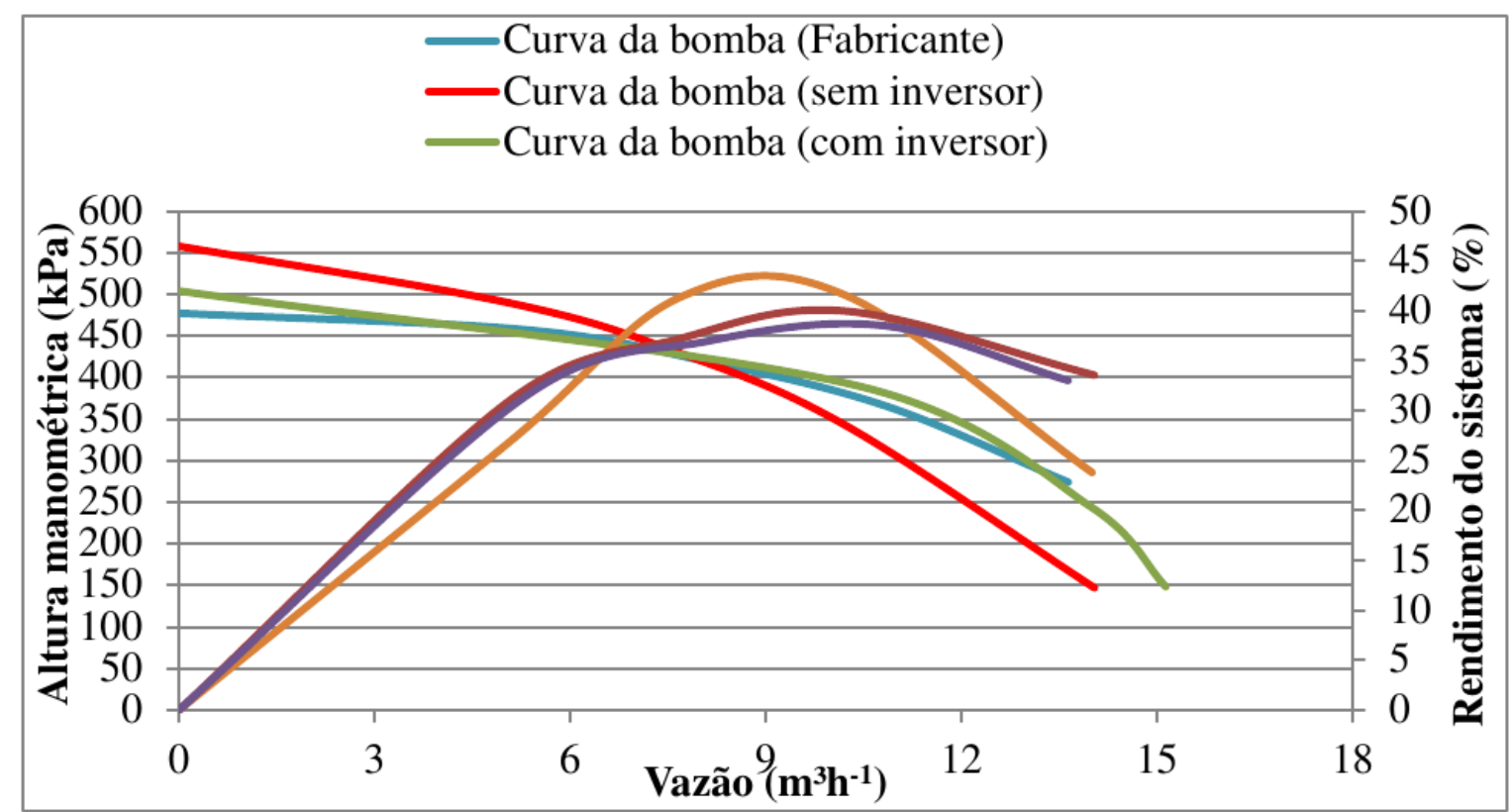

FIGURA 5. Curva característica da bomba ME 2230 2,2 kW (3 cv) fornecida pelo fabricante e rendimento do sistema sem e com inversor de frequência, obtidas em ensaio, em laboratório. Pump characteristic curve ME $22302.2 \mathrm{~kW}$ (3 hp) provided by the maker and system performance without and with frequency inverter, obtained in laboratory.

O rendimento dos motores elétricos foi determinado em função do índice de carregamento, razão entre a potência real e a nominal, e com este valor determina-se o rendimento do motor, o que pode ser verificado na Figura 6. Também pode determinar-se a corrente e o escorregamento, por meio de avaliação das curvas características do motor, fornecidas pelo fabricante.

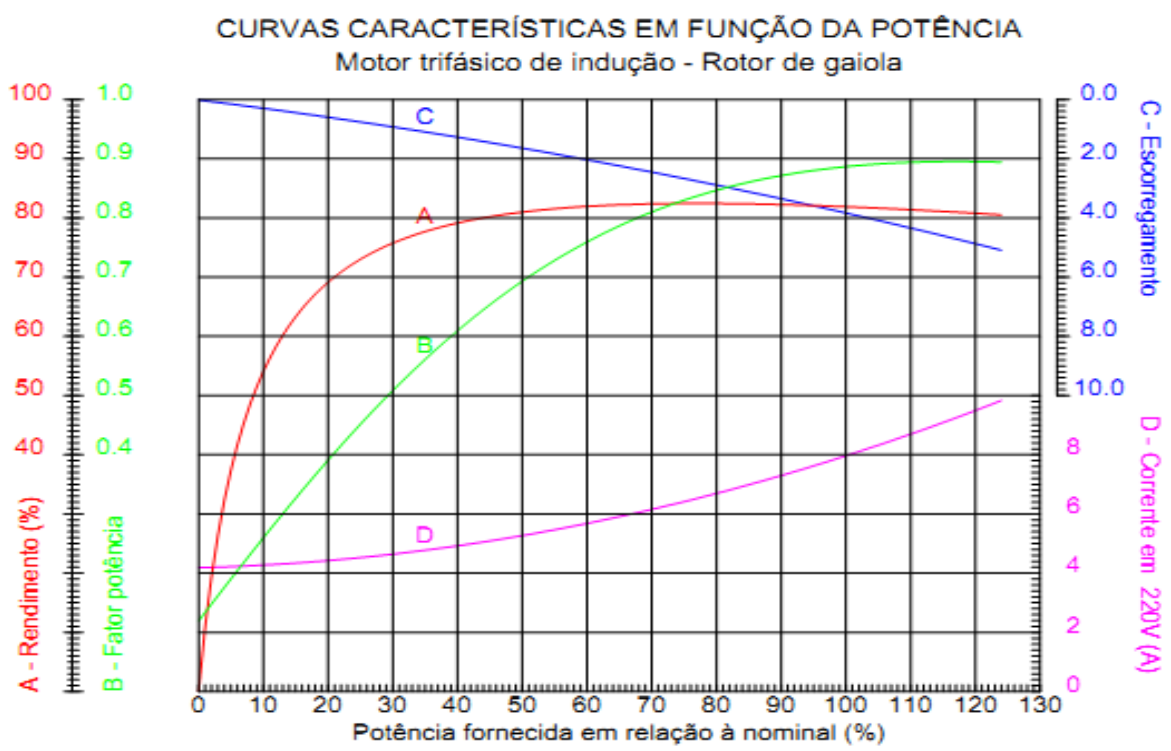

FIGURA 6. Curvas características do motor elétrico de $2,2 \mathrm{~kW}(3 \mathrm{cv})$. Characteristic curves of theelectric motor of $2.2 \mathrm{~kW}$ (3 hp) (WEG, 2012).

Como a altura manométrica sofreu variação para cada unidade de inclinação e unidade de rotação da linha lateral, calculou-se o índice de carregamento do motor elétrico para a declividade média de 30 \% em função da posição da linha lateral, que pode ser observado na Figura 7. 


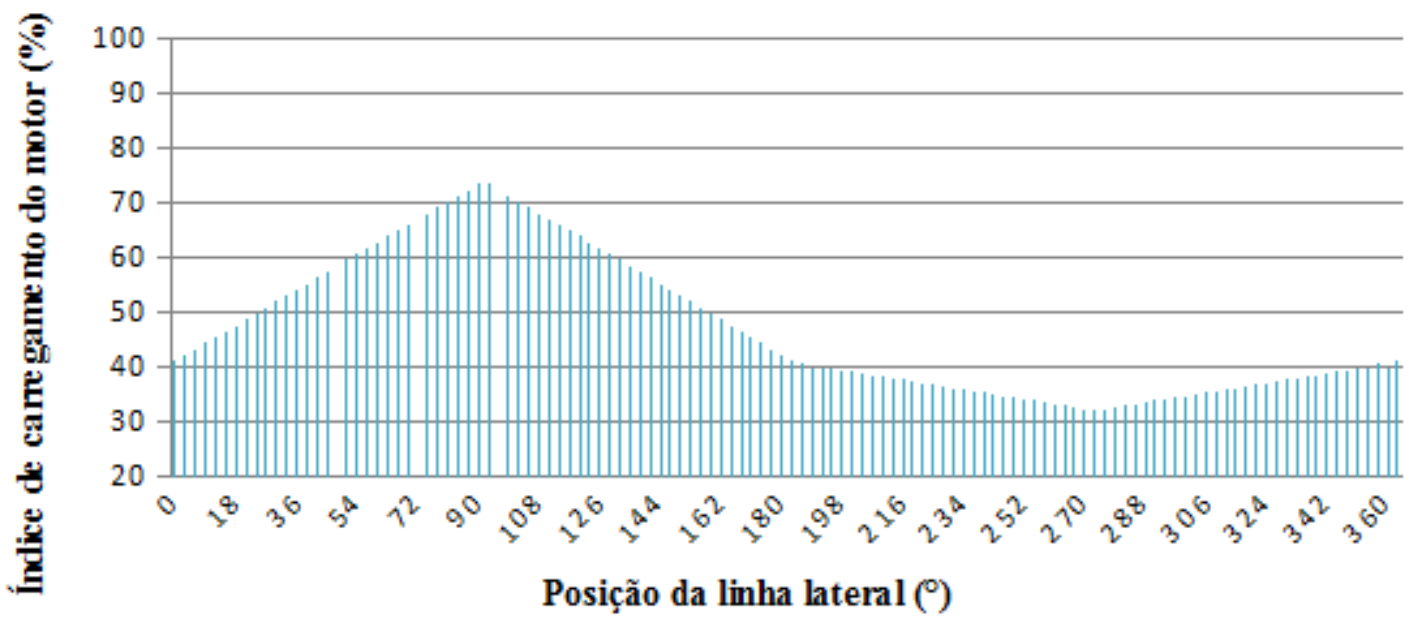

FIGURA 7. Índice de carregamento médio do motor sem inversor de frequência durante uma volta, em função da posição da linha lateral do pivô central, com declividade média de 30\%.

Average motor load without frequency inverter during a turn as a function of the lateral line of central pivot installed with $30 \%$ of average land slope.

Verificou-se que houve uma variação de potência fornecida em relação à nominal, à medida que o pivô central se desloca de um ponto de aclive para declive, e os valores variam de 73,2\% (30\% aclive) a 32,2 \% (30\% declive), necessitando de adequação de carga para cada unidade de rotação da linha lateral.

Como em todos os casos o índice de carregamento foi inferior a 75\%, observou-se a redução significativa do fator de potência e do rendimento.

A Figura 8 relaciona os requisitos de altura manométrica em função da posição do pivô central para a declividade de $30 \%$ para os seguintes casos: (i) desconsiderando a atuação das válvulas reguladoras de pressão, e (ii) considerando a atuação das válvulas reguladoras de pressão.

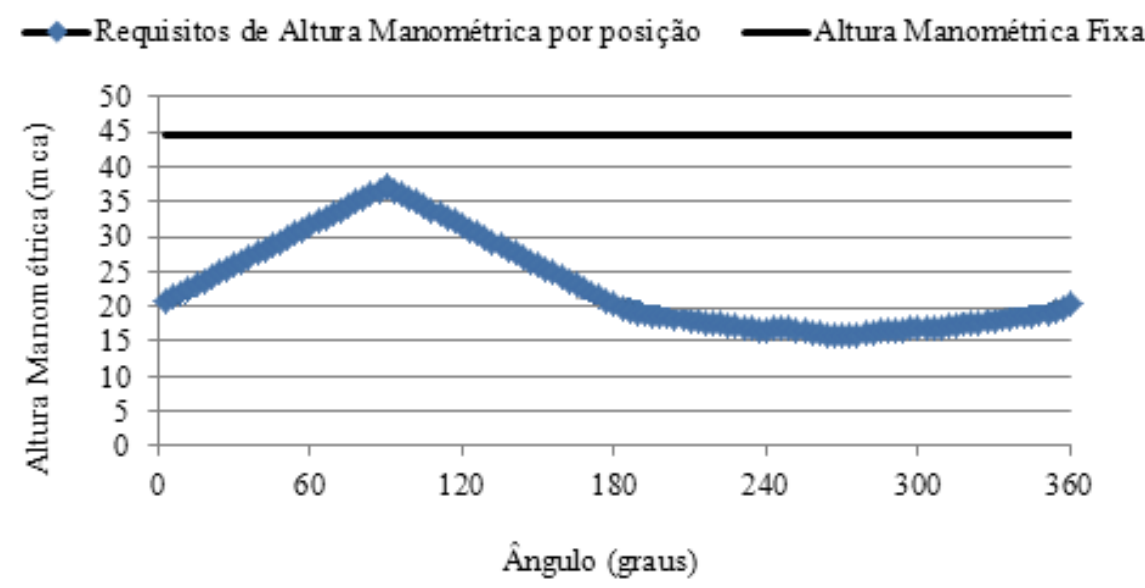

FIGURA 8. Altura manométrica em função do ângulo da linha lateral do pivô central para a declividade média de $30 \%$ quando se desconsidera a atuação das válvulas reguladoras de pressão. Pressure head as a function of the angle of the line of center pivot lateral for $30 \%$ average land slope when the pressure regulating valves action are disregarded . Fonte: (CAMPANA, 1999).

A Tabela 4 mostra os valores de pressão obtidos no protótipo para as declividades de 0,10 ; 20 e 30\% de declividade. Observa-se que a pressão no emissor 10 é superior à pressão de serviço $(167,19 \mathrm{kPa})$, estabelecida como limite de referência dos emissores. Este excesso de pressão estava em torno de 26; 41; 50 e 65\%, demandando maior consumo de energia pelo conjunto motobomba. 
TABELA 4. Pressão nos trechos da linha lateral do pivô usando o protótipo com controle eletromecânico, em função da vazão, comprimento de trechos, cota, frequência, rotação e potência consumida, para as declividades de 0; 10; 20 e 30\%. Pressure on the sideline sections of central pivot using the prototype with electromechanical control as a function of flow, sections' length, height, frequency, rotation and power consumed for land slopes of $0,10,20$ and $30 \%$.

\begin{tabular}{|c|c|c|c|c|c|c|c|c|c|c|c|}
\hline & & & & \multirow[b]{2}{*}{$0 \%$} & \multicolumn{7}{|c|}{ Declividade } \\
\hline & & & & & $10 \%$ & $20 \%$ & \multicolumn{4}{|c|}{$30 \%$} & \multirow[b]{2}{*}{ Pressão } \\
\hline $\begin{array}{l}\text { Trecho/ } \\
\text { emissor }\end{array}$ & $\begin{array}{l}\text { Compr. do } \\
\text { trecho }\end{array}$ & Vazão & $\begin{array}{l}\text { hf }(*) \\
(\mathrm{m})\end{array}$ & Cota & Pressão & Cota & Pressão & Cota & Pressão & Cota & \\
\hline & $(\mathrm{m})$ & $\left(m^{3} h^{-1}\right)$ & & (m) & $(\mathrm{kPa})$ & (m) & $(\mathrm{kPa})$ & $(\mathrm{m})$ & $(\mathrm{kPa})$ & $(\mathrm{m})$ & $(\mathrm{kPa})$ \\
\hline Pin & 6,00 & 7,63 & 0,23 & 0,00 & 406,70 & 0,00 & 406,70 & 0,00 & 406,70 & 0,00 & 406,70 \\
\hline 1 & 15,00 & 7,63 & 0,58 & 0,00 & 399,94 & 3,00 & 386,33 & 1,50 & 372,72 & 4,50 & 358,14 \\
\hline 2 & 4,60 & 7,28 & 0,16 & 0,00 & 398,97 & 3,92 & 380,50 & 1,96 & 363,00 & 5,88 & 341,61 \\
\hline 3 & 4,60 & 6,88 & 0,15 & 0,00 & 397,03 & 4,84 & 374,67 & 2,42 & 353,28 & 7,26 & 328,98 \\
\hline 4 & 4,60 & 6,37 & 0,13 & 0,00 & 396,06 & 5,76 & 368,83 & 2,88 & 343,56 & 8,64 & 309,53 \\
\hline 5 & 4,60 & 5,73 & 0,10 & 0,00 & 395,08 & 6,68 & 363,00 & 3,34 & 333,84 & 10,02 & 299,81 \\
\hline 6 & 4,60 & 2 & 0,08 & 0,00 & 394,11 & 7,60 & 358,14 & 3,80 & 324,12 & 11,40 & 283,28 \\
\hline 7 & 4,60 & 4,24 & 0,06 & 0,00 & 394,11 & 8,52 & 352,31 & 4,26 & 314,39 & 12,78 & 275,51 \\
\hline 8 & 4,60 & 3,31 & 0,04 & 0,00 & 393,14 & 9,44 & 347,45 & 4,72 & 304,67 & 14,16 & 256,06 \\
\hline 9 & 4,60 & 2,30 & 0,02 & 0,00 & 393,14 & 10,36 & 343,56 & 5,18 & 294,95 & 15,54 & 241,48 \\
\hline 10 & 4,60 & 1,19 & 0,01 & 0,00 & 393,14 & 11,28 & 338,70 & 5,64 & 283,28 & 16,92 & 226,90 \\
\hline \multicolumn{12}{|c|}{ Dados do motor elétrico } \\
\hline Rotação & (rpm) & & \multicolumn{3}{|c|}{3.476} & 3.473 & & \multicolumn{2}{|c|}{3.476} & \multicolumn{2}{|c|}{3.477} \\
\hline Frequência & $(\mathrm{Hz})$ & & \multicolumn{2}{|r|}{60} & & 60 & & \multicolumn{2}{|l|}{60} & \multicolumn{2}{|c|}{60} \\
\hline Potência & $(\mathrm{kW})$ & & \multicolumn{3}{|c|}{2,53} & 2,53 & & \multicolumn{2}{|c|}{2,53} & \multicolumn{2}{|c|}{2,53} \\
\hline
\end{tabular}

A Tabela 5 apresenta os valores de pressão nos emissores, rotação, frequência e o consumo de energia do sistema com inversor de frequência para 0; 10; 20 e 30 \% de declividade.

Verifica-se adequação de pressão próxima à pressão de serviço para o emissor 10. Observa-se que, para todas as declividades estudadas, os valores de frequência ótimos foram inferiores a $60 \mathrm{~Hz}$, e com isso houve redução na rotação e, consequentemente, redução do consumo de energia. 
TABELA 5. Pressão nos trechos da linha lateral do protótipo de pivô, em função da vazão, comprimento de trechos, cota, rotação, frequências e potência consumida, para as declividades de 0; 10; 20 e 30\% com inversor de frequência. Pressure on the sideline sections pivot using the prototype as a function of flow rate, length of sections, elevation, rotation, frequency and power consumption for land slopes of $0,10,20$ and $30 \%$ with frequency inverter.

\begin{tabular}{|c|c|c|c|c|c|c|c|c|c|c|c|}
\hline & & & & \multicolumn{8}{|c|}{ Declividade } \\
\hline & & & & $0 \%$ & $10 \%$ & $20 \%$ & $30 \%$ & & & & \\
\hline Trecho & Comp. & Vazão & $\begin{array}{l}\text { Perda } \\
\text { carga }\end{array}$ & Cota & Pressão & Cota & Pressão & Cota & Pressão & Cota & Pressão \\
\hline & (m) & $\left(\mathrm{m}^{3} \mathrm{~h}-1\right)$ & $(\mathrm{m})$ & (m) & $(\mathrm{kPa})$ & (m) & $(\mathrm{kPa})$ & (m) & $(\mathrm{kPa})$ & $(\mathrm{m})$ & $(\mathrm{kPa})$ \\
\hline $\mathrm{P}_{\text {in }}$ & 6,00 & 7,63 & 0,23 & 0,00 & 198,03 & 0,00 & 252,56 & 0,00 & 307,10 & 0,00 & 371,56 \\
\hline 1 & 15,00 & 7,63 & 0,58 & 4,50 & 178,29 & 3,00 & 222,04 & 1,50 & 256,06 & 0,00 & 294,95 \\
\hline 2 & 4,60 & 7,28 & 0,16 & 5,88 & 171,49 & 3,92 & 207,46 & 1,96 & 246,34 & 0,00 & 280,37 \\
\hline 3 & 4,60 & 6,88 & 0,15 & 7,26 & 170,51 & 4,84 & 197,73 & 2,42 & 236,62 & 0,00 & 270,65 \\
\hline 4 & 4,60 & 6,37 & 0,13 & 8,64 & 169,54 & 5,76 & 192,87 & 2,88 & 226,90 & 0,00 & 256,06 \\
\hline 5 & 4,60 & 5,73 & 0,10 & 10,02 & 169,54 & 6,68 & 188,01 & 3,34 & 210,37 & 0,00 & 236,62 \\
\hline 6 & 4,60 & 5,02 & 0,08 & 11,40 & 168,57 & 7,60 & 178,29 & 3,80 & 205,51 & 0,00 & 217,18 \\
\hline 7 & 4,60 & 4,24 & 0,06 & 12,78 & 168,57 & 8,52 & 173,43 & 4,26 & 197,73 & 0,00 & 212,32 \\
\hline 8 & 4,60 & 3,31 & 0,04 & 14,16 & 168,57 & 9,44 & 170,51 & 4,72 & 186,07 & 0,00 & 192,87 \\
\hline 9 & 4,60 & 2,30 & 0,02 & 15,54 & 168,57 & 10,36 & 168,57 & 5,18 & 178,29 & 0,00 & 183,15 \\
\hline 10 & 4,60 & 1,19 & 0,01 & 16,92 & 168,57 & 11,28 & 168,57 & 5,64 & 168,57 & 0,00 & 168,57 \\
\hline \multicolumn{12}{|c|}{ Dados do motor elétrico } \\
\hline Rotação & (rpm) & & 3.276 & 3.078 & 2.865 & \multicolumn{6}{|c|}{2.661} \\
\hline Frequência & $(\mathrm{Hz})$ & & 56,3 & 53,0 & 49,5 & \multicolumn{6}{|c|}{46,0} \\
\hline Potência & $(\mathrm{kW})$ & & 2,11 & 1,87 & 1,57 & \multicolumn{6}{|c|}{1,31} \\
\hline
\end{tabular}

Para cálculo de consumo de energia elétrica, foi considerado o tempo de 7 horas para a linha lateral percorre de 0 a $180^{\circ}$, e os resultados estão ilustrados na Figura 9, que demonstra que a utilização do inversor proporcionou uma economia de energia elétrica de 48 a $16 \%$.

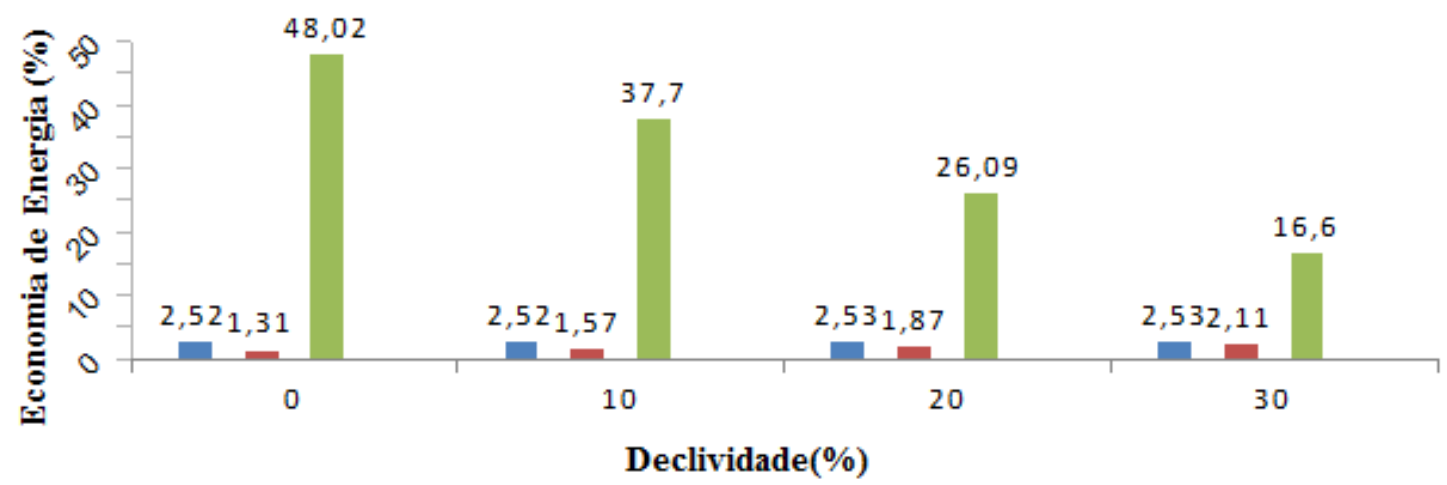

\footnotetext{
- Potência consumida com controle eletromêcanico, kW

- Potência consumida com controle com inversor de frequência sem retroalimentação, kW

Economia de energia usando os dois sistemas de controle, \%
}

FIGURA 9. Relação entre a potência elétrica medida antes e após a variação de rotação e o respectivo potencial de economia de energia para diferentes declividades. Relationship among the electrical power measured before and after the rotation variation and their potential energy savings for different land slopes.

Comparando-se a variação da velocidade de rotação e o rendimento dos motores acoplados a inversores, os fabricantes de motores, em seus catálogos técnicos, fazem algumas considerações em 
relação ao rendimento do conjunto motor-inversor de frequência, como segue: O motor de indução, quando alimentado por um inversor de frequência PWM, tem seu rendimento diminuído, em relação a um motor alimentado por tensão puramente senoidal, devido ao aumento nas perdas ocasionadas por componentes harmônicas: (I) Em aplicações de motores com inversores, deve ser avaliado o rendimento do sistema (inversor-motor) e não apenas do motor. (II) Devem ser consideradas as características do inversor do motor, tais como frequência de operação, frequência de chaveamento, condição de carga e potência do motor, taxa de distorção harmônica do inversor, entre outros. (III) Instrumentos especiais, capazes de medir o valor eficaz verdadeiro (true RMS) das grandezas elétricas, devem ser utilizados. (IV) $\mathrm{O}$ aumento da frequência de chaveamento diminui o rendimento do inversor e aumenta o rendimento do motor.

Com a averiguação do potencial da utilização do inversor de frequência, foi desenvolvido um sistema de controle de velocidade do conjunto motobomba a fim de que o sistema consiga atender à pressão mínima nos emissores com menor rotação do conjunto motobomba, impactando na redução do consumo de energia elétrica. Como o sistema em laboratório permitia somente a verificação de aclive (de $0^{\circ}$ a $90^{\circ}$ ), então o ponto crítico seria o emissor 10 , pois ele era o mais afastado e com maior cota em relação à bomba. Quando a pressão no emissor 10 estava satisfatória, por conseguinte, todos os outros emissores estavam com pressão superior à deste emissor, atendendo às pressões de serviço demandadas pelo do sistema de irrigação.

Verificou-se que o sistema funcionou de forma adequada, e seu tempo de reação foi de aproximadamente 20 segundos, para ler a pressão existente no ponto 10 e enviar um sinal ao motor de passo no controle do inversor.

Verificou-se, na Figura 9, que no protótipo automatizado, houve redução da potência consumida, e isso ocorreu devido ao sensor ser mais sensível para leitura que o manômetro utilizado. Desta forma, verificou-se a necessidade de melhor aferição do transdutor de pressão.

\section{CONCLUSÕES}

O protótipo de pivô central mostrou ser capaz de simular condição real, e com linha lateral inclinada em 0; 10; 20 e 30\%, verificou-se que o uso do inversor de frequência proporcionou uma economia de energia elétrica de aproximadamente 48; 37; 26 e 16 \%, respectivamente. A automação do pivô central usando o inversor de frequência mostrou-se viável, eficaz e capaz realizar a variação da rotação conforme a pressão estabelecida como necessário nos pontos considerados críticos.

\section{AGRADECIMENTOS}

Os autores são gratos pelo apoio da FAPEMIG, CAPES, CNPq, a Universidade Federal de Viçosa e a Universidade Estadual de Goiás.

\section{REFERÊNCIAS}

BERNARDO, S.; SOARES, A. A.; MANTOVANI, E. C. Manual de irrigação. 8.ed. Viçosa: UFV, 2006. p. 625.

CAMPANA, S.; OLIVEIRA FILHO, D.; SOARES, A. A. Racionalização do Uso de Força Motriz em Sistema de Irrigação do Tipo Pivô Central e Aspersão Convencional. Engenharia na Agricultura (Impresso), v. 7, p. 30-50, 1999.

FERNANDES, A. L. T.; TESTEZLAF, R. Fertirrigação na cultura do melão em ambiente protegido, utilizando-se fertilizantes organo-minerais e químicos. Revista Brasileira de Engenharia Agrícola e Ambiental, Campina Grande, v. 6, n. 1, p. 45-50, 2002

JIMÉNEZ-BELLO, M. Á., ALZAMORA, F. M., CASTEL, J. R., INTRIGLIOLO, D. S. Validation of a methodology for grouping intakes of pressurized irrigation networks into sectors to minimize energy consumption. Agricultural Water Management, Amsterdam, n.102, p. 46-53,2011. 
MORENO, M. A., CÓRCOLES, J. I., TARJUEL, J. M., ORTEGA, J. F. Energy efficiency of pressurized irrigation networks managed on-demand and under a rotation schedule. Biosystems Engineering, London, p. 349-363, 2010.

MOUSAVI-AVVAL, S. H., RAFIEE, S., JAFARI, A., MOHAMMADI, A. Optimization of energy consumption for soybean production using Data Envelopment Analysis (DEA) approach. Applied Energy, London, n. 88, p.3765-3772, 2011.

OLIVEIRA FILHO, D.; SAMPAIO, R. P.; MORAES, M. J.; PIZZIOLO, T. A.; DAMIÃO, J. H. A. C. Metodologia de diagnóstico energético em estação de captação de água. Revista Brasileira de Engenharia Agrícola e Ambiental. v.15, n.10, p. 1097-1103, 2011.

RIBEIRO, M. C., Eficientização e gerenciamento do uso de energia elétrica em perímetros irrigados. 2008. 178f. Tese (Doutorado em Engenharia Agrícola) - Universidade Federal de Viçosa, Viçosa, 2011.

SANTOS, C.; LORITE, I.J.; TASUMI, M.; ALLEN, R.G.; FERERES, E. Performance of an irrigation scheme using indicators determined with remote sensing techniques. Irrigation Science, New York, n.28, p.461-477, 2010.

SUDHA, M.N., VALARMATHI, M. L., BABU, A. S. Energy efficient data transmission in automatic irrigation system using wireless sensor networks. Computers and Electronics in Agriculture, New York, p. 215-221, 2011.

VIANA, A.N.C.; WERDINE, D.; BORTONI, E.C.; HADDAD, J.; SOUZA, P.R.C.;

BERENHAUSER, C.J.T. Uso eficiente de energia elétrica e conservação de água em sistemas de saneamento ambiental. Rio de Janeiro: DIEE/Eletrobrás/PROCEL, 2004. 348

VIEIRA, G. H. S. ; MANTOVANI, E.C. ; SOARES, A. A. ; MONTES, D.R.P. ; CUNHA, F. F. Custo da irrigação do cafeeiro em diferentes tipos de equipamento e tamanhos de área. Engenharia na Agricultura, Viçosa, MG, v. 19, p. 53-61, 2011.

WEG. Catálogo eletrônico. Disponível em: http://www.weg.net/. Acesso em 10 jan 2013. 